ABSTRACT

\title{
Summary of NASA Advanced Telescope and Observatory Capability Roadmap
}

\author{
H. Philip Stahl, NASA MSFC \\ Lee Feinberg, NASA GSFC
}

The NASA Advanced Telescope and Observatory (ATO) Capability Roadmap addresses technologies necessary for NASA to enable future space telescopes and observatories operating in all electromagnetic bands, from x-rays to millimeter waves, and including gravity-waves. It lists capability priorities derived from current and developing Space Missions Directorate (SMD) strategic roadmaps. Technology topics include optics; wavefront sensing and control and interferometry; distributed and advanced spacecraft systems; cryogenic and thermal control systems; large precision structure for observatories; and the infrastructure essential to future space telescopes and observatories. 


\section{Summary of NASA Advanced Telescope and Observatory Capability Roadmap}

\author{
H. Philip Stahl \\ NASA MSFC XD-30 \\ Huntsville, AL 35812 \\ (256) $544-0445$ \\ h.philin stanlonasa.gov
}

\author{
Lee Feinberg \\ NASA GSFC \\ Greenbelt, MD 20771 \\ (301) 286-5923 \\ LFeinbergonstmasa.gov
}

\begin{abstract}
The NASA Advanced Telescope and Observatory (ATO) Capability Roadmap addresses technologies necessary for NASA to enable future space telescopes and observatories operating in all electromagnetic bands, from $x$-rays to millimeter waves, and including gravity-waves. It lists capability priorities derived from current and developing Space Missions Directorate (SMD) strategic roadmaps. Technology topics include optics; wavefront sensing and control and interferometry; distributed and advanced spacecraft systems; cryogenic and thermal control systems; large precision structure for observatories; and the infrastructure essential to future space telescopes and observatories.
\end{abstract}

\section{TABLE OF CONTENTS}

\section{INTRODUCTION}

2. ADVANCED TELESCOPE AND OBSERVATORY CAPABILITY ROADMAP

3. SUMMARY OF SUB-CAPABILITIES

4. CAPABILITY ROADMAP TIMELINES

5. SUMMARY

REFERENCES

BIOGRAPHY

\section{INTRODUCTION}

On January 14, 2004, President George W. Bush set the nation's space program in a new direction with the Vision for Space Exploration (Vision). The fundamental goal of the Vision is to advance the scientific, security, and economic interests of the United States via a robust space exploration program.

In February 2005, NASA defined eighteen Strategic Objective in The New Age of Exploration NASA's Direction for 2005 and Beyond. Four objectives were determined to require advanced telescopes and observatory technology:

\#4 Conduct Advanced telescope searches for Earth-like planets and habitable environments around the stars.

\#5 Explore the Universe to understand its origin, structure, evolution and destiny.

\footnotetext{
1 "U.S. Government work not protected by U.S. copyright."

${ }^{2}$ IEEEAC paper \# 1187, Version 3, Updated 8 December 2006.
}

\#14 Advance scientific knowledge of the Earth system through space-based observation, assimilation of new observations, and development and deployment of enabling technologies, systems, and capabilities, including those with the potential to improve future operational systems.

\#15 Explore the Sun-Earth System to understand the Sun and its effects on Earth, the solar system, and the space environmental conditions that will be experienced by human explorers, and demonstrate technologies that can improve future operational systems.

The President's "Commission on Implementation of United States Space Exploration Policy". (The Aldridge Commission) was chartered to prepare recommendations for implementing the Vision. In response to this commission's report, NASA established roadmap teams to recommend strategic and capability priorities, options and alternatives, technology strategies, and other key elements necessary to achieve the Vision.

NASA's Advanced Planning and Integration Office (APIO) chartered thirteen strategic roadmap (SRM) teams to explore options and establish pathways for implementing the Vision. The four SRM teams which require advanced telescope and observatory technology are:

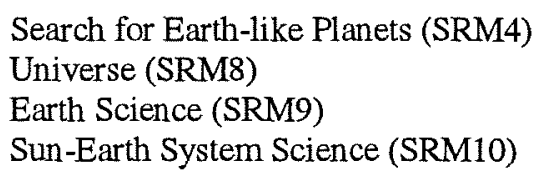

APIO also commissioned fifteen Capability Roadmap (CRM) teams to identify the technical knowledge and expertise required by the Agency to implement the strategic roadmaps and to identify the types of technology and capability investments that the Agency needs to make to achieve NASA's highest priorities. The CRM teams identified and analyzed technologies and technical challenges, assessed the current state of the art, estimated the development time to achieve capabilities, and identified key architectural and strategic decisions that would affect the direction of the strategic roadmaps.

The Advanced Telescope and Observatory (ATO) CRM was one of these teams. This paper is extracted from the NASA Capability Roadmaps Executive Summary, 22 May 2005. 


\section{ADVANCED TELESCOPE AND OBSERVATORY CAPABILITY ROADMAP}

The Advanced Telescope and Observatory (ATO) Roadmap Committee was chartered to examine technologies necessary to enable future space telescopes and observatories operating in electromagnetic bands, ranging from $\mathrm{x}$-rays to millimeter waves, and also gravity-waves.

An underlying philosophy for the roadmap development was to include participants from across the nation. The roadmap teams were comprised of technical experts from academia, industry, NASA, and other Government Agencies. Each team was co-chaired by a NASA and nonNASA subject matter expert. The ATO membership was comprised approximately of $1 / 3$ each of Government, Industry and Academic technical experts (Table 1). This structure was meant to ensure a national perspective and mitigate institutional biases.

Table 1. ATO Committee Participants

\section{Government}

Lee Feinberg, NASA (Chair)

Jim Breckinridge, Jet Propulsion Laboratory

Pete Jones; Air Force Research Laboratory

David Tratt, Jet Propulsión Laboratory

H. Philip Stahl, NASA

\section{Industry}

Howard MacEwen, SRS Technologies (Co-Chair)

Jim Crocker, Lockheed Martin

Ron Polidan, Northrop Grumman Space Technology

Gary Matthews, ITT Aerospace

Mark Stier, Goodrich Aerospace

Jim Oschmann, Ball Aerospace

\section{Academia}

Jim Fienup, University of Rochester

Dave Miller, Massachusetts Institute of Technology

Jim Burge, University of Arizona

Dan Inman, Virginia Technology

For planning purposes, the ATO team assumed a list of missions and launch dates provided by APIO (Table 2).

\section{Table 2. Assumed ATO Mission List}

James Webb Space Telescope (JWST)

Space Interferometer Mission (SIM)

Laser Interferometer Space Antenna (LISA)

Tropical Wind LIDAR

Terrestrial Planet Finder Coronagraph (TPF-C) 2016

Geospace Electrodynamics Connections (GEC) 2016

Constellation X (ConX) 2017

Terrestrial Planet Finder Interferometer (TPF-I) 2019

Large Microwave

Magnetic Constellation

2021

Inflation Probe (IP)
Single Aperture Far-Infrared (SAFIR)

GEO LIDAR

Large Ultra-Violet Observatory (LUVO)

GEO Surface Deformation

Life Finder (LF)

Black Hole Imager (BHI)

Big Bang Observer (BBO)

2028

Stellar Imager (SI)

Far-Infrared Sub-MM Interferometer (FIRSI)

2030

2032

Planet Imager (PI)

This list was compared to the recent National Academy of Sciences Astronomy and Astrophysics Decadal Survey and verified through dialog with Strategic Roadmap panels.

JWST and SIM were included for reference only. They are not part of the roadmap because they are currently in Phase B development. Probe, Explorer and Discovery class missions (such as the Dark Energy Mission) are not called out in the roadmap for several reasons. First, the selection of such missions for flight is typically competed and thus cannot be scheduled. Second, while such missions might benefit from technology advances, they do not require any enabling technology. Other missions (such as Early Universe X-Ray Observer, EUXO) were not included because their potential launch date is beyond 2035 .

The ATO team derived capability needs to enable and enhance these missions from NASA heritage, current and developing Science Mission Directorate (SMD) strategic roadmaps. Additionally, each mission made presentations and provided reference material: To insure that capabilities were not missed, ATO collaborated closely with other teams, particularly the Scientific Instruments and Sensors Roadmap team, which had the responsibility to address technologies associated with the detection, conversion, and processing of observed signals into data.

The ATO roadmap is organized around a Capability Breakdown Structure (Figure 1). The roadmap consists of six basic capabilities (each of which is further broken down into sub-capabilities): Optics, Wavefront Sensing and Control, Distributed and Advanced Spacecraft Systems, Large Precision Structures, Cryogenic and Thermal Control Systems, and Infrastructure. Potential capability overlaps with other CRM teams were resolved. The Scientific Instruments and Sensors roadmap was assumed to treat heat pipe cooling to radiators, optical bench cooling, detector cooling, instrument optics, microwave system electronics and antennas/waveguides, and laser systems. The modeling roadmap committee was assumed to cover modeling and integrated modeling tools.

Once the capability requirements were defined, ATO surveyed the current state-of-art and investment strategies of multiple NASA Centers, Government Agencies, Industries and Universities. Additionally, ATO accepted public input during an APIO sponsored workshop. 


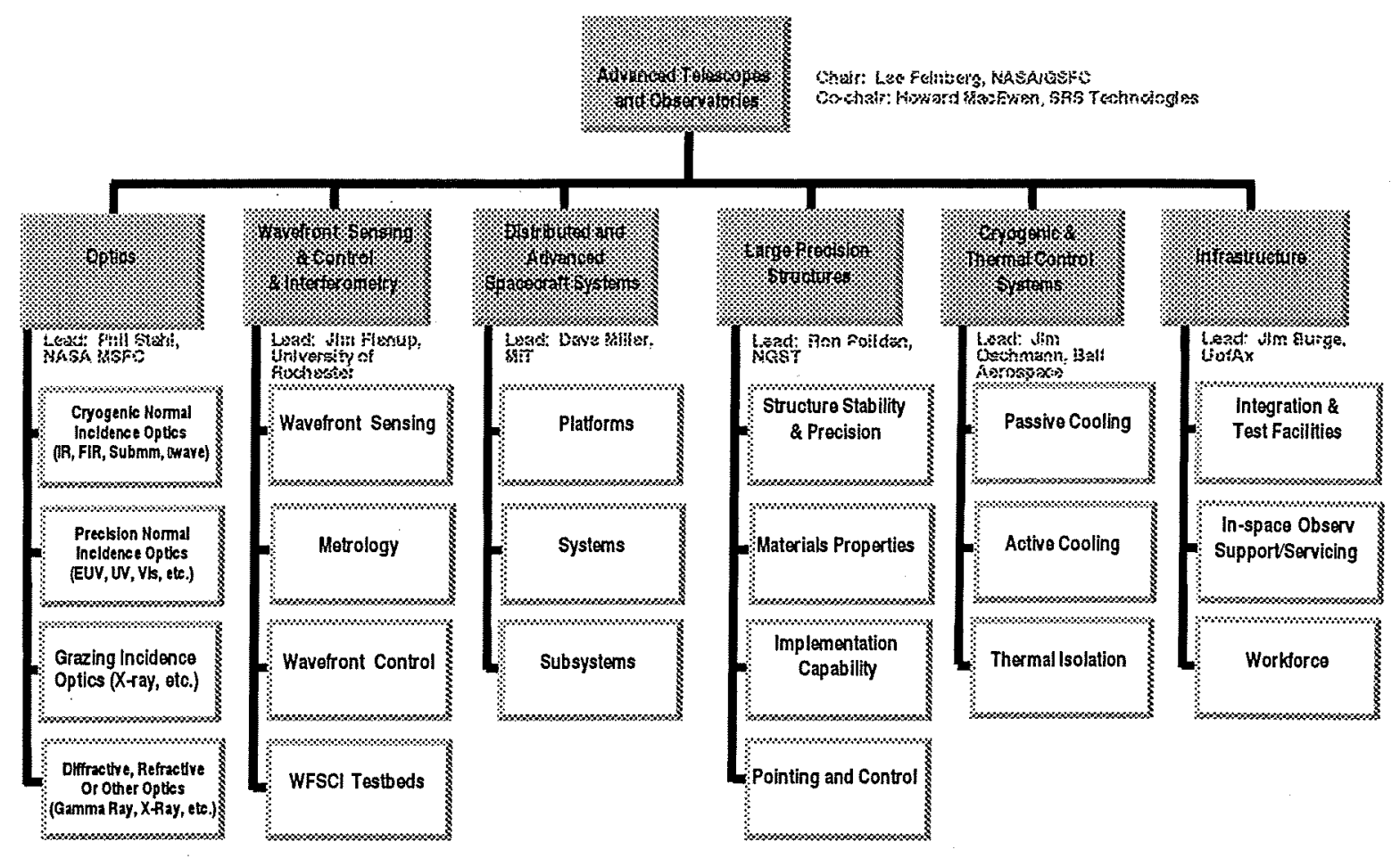

Figure 1 Advanced Telescope and Observatory Capability Breakdown Structure

Finally, the ATO team presented its Roadmap to the National Research Council for review and comment on 15 March 2005.

\section{SUMMARY OF SUB-CAPABILITIES}

\subsection{OPTICS}

Lightweight affordable optics is an enabling capability for future large-aperture space optical systems for Earth science, solar observations, and astronomy. The ATO CRM defines an optics capability as a system of components such as mirror substrates and facesheets, coatings, and actuators, along with the respective manufacture and test processes necessary to collect and concentrate electromagnetic radiation. The roadmap further defines four sub-capabilities based principally upon wavelength region:

- Cryogenic Optics (IR, Far-IR, Sub-mm, Microwave)

- Precision Optics (EUV, FUV, UV, Visible, LIDAR)

- Grazing Incidence Optics (FUV and X-Ray)

- Diffractive, Refractive, and Novel Optics (Gamma, $\mathrm{X}$ - ray or other)
Associated with each sub-capability are many technical figures of merit that directly map into system technical performance parameters. The study for the ATO CRM considered four: mirror surface figure error (or resolution for X-ray mirrors), areal density, size and areal cost.

Regardless of the operating wavelength or mission application, the greatest technical challenge for optics is the ability to make large-aperture low-areal-density mirrors of sufficient surface figure precision, surface finish, and mechanical stiffness. Current observatories are mass and volume limited due to the launch vehicle, which in turn limits the maximum attainable aperture. Developing a capability to produce lower areal density mirrors with efficient launch packaging and deployment concepts will enable future large aperture observatories. Furthermore, lightweight optics must be very stiff and thermally stable to retain the required optical figure and accurate, stable line of sight pointing. Regardless of the operating wavelength or application, the greatest programmatic challenge for large space optics is to rapidly manufacture affordable mirrors. Reducing the areal cost (dollars per square meter) of mirrors enables the acquisition of systems with larger apertures that will still fit within the constraints of launch mass and volume limits.

While these technologies are common requirements for optical systems at all wavelengths, other technological capabilities can be equally significant over specific wavebands. 


\section{Cryogenic Optics:}

Future infrared/far-infrared/sub-millimeter and millimeter wavelength missions require very large aperture but modestquality mirrors operating at temperatures from 4 to $40 \mathrm{~K}$ to reduce background noise. Current state of the art cryogenic mirrors can satisfy most of the technical requirements for such missions, but the cost is too great. Thus, for this waveband, the most important enabling capability is to reduce their areal cost by an order of magnitude. Approaches to achieve this goal include replication, nanolaminates, near-net shaping and advanced polishing techniques. Another specific enabling technology of particular note in this spectral region is polarization preserving uniform coatings.

\section{Precision Optics:}

Future extreme ultraviolet, ultraviolet and visible wavelength missions will require large-aperture, extremely smooth, and highly stable ambient temperature mirrors. The most challenging mission in the near term is TPF-C (Terrestrial Planet Finder Coronagraph). TPF-C requires a primary mirror that has never before been demonstrated on the ground let alone in space - an extremely smooth $(4 \mathrm{~nm}$ $\mathrm{rms}$ surface) 4 by 8 meter lightweight $\left(\sim 40 \mathrm{~kg} / \mathrm{m}^{2}\right)$ mirror with extremely uniform optical coating reflectivity and polarization properties. The cost-effective fabrication of such a mirror requires the application to an 8 meter class mirror of precision optical metrology techniques previously only demonstrated on $0.5 \mathrm{~m}$ class microlithography optics.

Because of launch vehicle limitations, some future missions may choose a segmented mirror architecture. While it is easier to manufacture smaller mirror segments, a segmented mirror telescope operating in the UV/Visible has its own challenges. To minimize scattered light and diffraction effects, the segments must be accurately figured and polished completely to the mirror's physical edge. Additionally, each segment's position must be mechanically controlled to extreme tolerances $(0.1 \mathrm{~nm})$. Three specific enabling coating technologies are $80 \%$ reflectivity coatings from 90 to $120 \mathrm{~nm}, 0.1 \%$ uniform reflectivity and $0.1 \%$ uniform polarization coatings from 400 to $1000 \mathrm{~nm}$, and improved dichroic, spectral and combiner coatings.

\section{Grazing Incidence Optics:}

Future $\mathrm{x}$-ray and far-ultra-violet missions require largeaperture precision-quality grazing incidence mirrors. The technology required to produce these mirrors is truly revolutionary when compared to Chandra. The cost cap and mass/volume limitations of grazing optics are profound when compared with normal incidence optics. Doubling the collecting area of a grazing incidence telescope can require as much as a $400 \mathrm{X}$ increase in actual mirror surface area. For example, the Constellation- $X$ mission plans a four telescope architecture with $60 \mathrm{X}$ the effective collecting aperture as Chandra (6 square meters). Each telescope is planned to be 1.6 meter diameter $\mathrm{x} 1$ meter long with $20 \mathrm{X}$ lower areal density $(<3 \mathrm{~kg} / \mathrm{m} 2)$ and $50 \mathrm{X}$ lower areal cost $(<\$ 0.1 \mathrm{M} / \mathrm{m} 2)$ than Chandra. The technology needed to manufacture these mirrors requires developing new materials and fabrication processes. Obviously, mass production via some type of replication process is a leading candidate. The only mitigating factor is that, at 15 arcsecond resolution, ConX has $30 \mathrm{X}$ looser optical surface figure error requirements than Chandra. However, because of the lower areal density, the mechanical support, alignment and stability of such optics are a significant challenge. And, the technical challenges only increase for envisioned missions Black Hole Imager (BHI) and Extreme Universe X-Ray Observatory (EUXO).

Additionally, there is also an on-going need to invest in normal incidence $x$-ray optics technology. This technology is needed to support a series of smaller scale Sun-Earth science missions that require $\mathrm{x}$-ray optics and which could benefit from incremental quality and cost reduction improvements.

\section{Diffractive, Refractive and Novel Optics:}

In addition to the areas discussed in the preceding sections, there is also a need for diffractive, refractive and novel optics that includes coded apertures, occulting imaging, holographic optical elements (HOEs), Fresnel lènses, etc. These classes of novel optics are hard to roadmap because of their early stage of development, but they may enable enhanced (and more affordable) approaches to planned missions as well as unexpected missions through their clever use of novel concepts in optics. This is a critical area to encourage, particularly as the technological challenges increase in difficulty for traditional optics approaches.

\subsection{WAVEFRONT SENSING AND CONTROL AND INTERFEROMETRY}

Most future missions require increasingly larger apertures to collect faint light from distant and cold sources and to provide high angular resolution for investigating the "fine structure" of the universe. One solution is a large monolithic telescope. But, to make one light enough to launch in current rockets, its stiffness may be inadequate to passively maintain the required high quality wavefront. In such cases, active wavefront sensing and control (WFSC) can be used to compensate for fabrication and on-orbit wavefront errors.

Another approach is spatial interferometry which divides a very large aperture telescope into separate smaller, discrete apertures. Extremely high angular resolution is enabled by combining these smaller aperture telescopes across areas larger than can be covered by a single aperture, in some cases so large that the separate telescopes can no longer be structurally connected, but instead must be flown separately and use WFSC to create a large synthetic aperture. 
Both single-aperture telescopes and interferometers require new wavefront sensing and control technology. WFSC is a system-level technology that includes sensors to characterize a reference source (in some cases, the source itself must be provided), signal processing, real-time computation of control signals for opto-mechanical devices and actuators, and distributed system communication to the mechanical control system. WFSC reference sources may be artificial or natural, and include lasers, edge sensors on mirror segments, or a sufficiently strong source in the field of view. Two critical components of WFSC systems in particular require dedicated attention for future space telescope systems are wavefront sensors and actuators.

Several potential future missions illustrate the criticality of advanced, high precision WFSC. For example, TPF-C needs to sense and correct wavefronts with two orders of magnitude greater accuracy than JWST. One way to achieve this control is with 50 picometer $(\mathrm{pm})(\lambda 10,000)$ deformable mirrors which are stable over periods of hours. TPF-C also needs speckle-suppression hardware and software to achieve the required $10^{10}$ contrast in broadband light. A possible Large UV-Optical telescope (LUVO), with its shorter wavelengths in the visible and ultraviolet, will require five times better WFSC ( $8 \mathrm{~nm}$ RMS) than JWST and may be required to operate continuously in an autonomous, closed-loop fashion: Formation-flying systems, such as the Terrestrial Planet Finder Interferometer (TPF-I), a stellar imager, and life finder will not be possible without advanced WFSC.

Ground-based testbeds have been and will continue to be essential for developing the ability to sense and control wavefronts under realistic conditions and for validating algorithms and models. Several WFSC testbeds were developed for both JWST and SIM, and have been in active use for several years. New missions will require increasingly complex test beds. Technology is needed to better calculate and emulate the space environment $(0 \mathrm{~g}$, radiation field, thermal background, and space contamination). Fundamental research is needed in algorithm development, high speed digital signal processing, actuator devices, low power devices, long lifetime lasers, and advanced sensors.

\subsection{DISTRIBUTED AND ADVANCED SPACECRAFT SYSTEMS}

A distributed spacecraft system is a set of two or more spacecraft whose dynamics are coupled through a cooperative sensing and control architecture. This enables a distributed network of individual vehicles to act collaboratively as a single functional unit that can exhibit a common system wide capability. Such a capability holds the promise of revolutionizing space-based astronomy. By extending the modularity inherent in sparse aperture optical systems to the supporting spacecraft, new operational modalities emerge. Formation flight of optical sub- apertures enables angular resolution far beyond that which is attainable with structurally connected arrays, the tuning of the point spread function to the object being observed, and the synthesis (through maneuvers) of images that would otherwise require prohibitively large filled apertures. Furthermore, distributed systems enable reconfiguration of the array in the event of spacecraft failure and the ability to add new spacecraft to the formation. Finally, with a rendezvous and docking capability, cryostats can be replenished, spacecraft can be refueled, and detectors can be upgraded. High packaging efficiency during launch can be achieved through the use of modular components that are deployed or robotically assembled on orbit.

The major challenge to implementing distributed system architectures is the need to affordably fabricate multiple identical spacecraft within a reasonable schedule. Another key issue is propulsion. Reactive propulsion technology used to maneuver and maintain formation, limits mission lifetime through depleting consumables and contaminating the spacecraft environment (deposition on optical surfaces, plume impingement, thermal emission, etc.). Therefore, propellant-less formation flight should be investigated including the use of carefully designed orbital dynamics, tethers, natural fields (magnetic, solar pressure), and artificial fields generated by the spacecraft themselves (electro-magnetic, electrostatic).

As shown in Figure 2, many planned Earth and space science missions have baselined distributed, formationflying architectures. Yet, no-mission has flown to test the duration, precision, autonomy, reconfigurability, or number of spacecraft needed for these missions. Since multiple challenging capabilities must be matured to enable precision spacecraft formation flight, distributed spacecraft systems would benefit from a reconfigurable test platform where technology "layers" can mature in a spiral development: first maturing algorithms in a risk-tolerant setting; then maturing spacecraft sub-systems such as propulsion, sensing, and communications; and finally demonstrating payload technologies including collectors, combiners and optical control. Such a test sequence could be based upon the internal and external test environments provided by the International Space Station (ISS).

\subsection{LARGE PRECISION STRUCTURES FOR OBSERVATORIES}

Developing new capabilities to affordably produce and deploy large precision structures for future observatories is an enabling technology for the majority of space and Earth science missions, for which aperture size is a critical factor. Increased aperture creates greater sensitivity and greater resolution across the entire electromagnetic spectrum. Such structures are a capability being developed for the first time with the James Webb Space Telescope (JWST). JWST already exceeds the volume capability of current launch vehicles: it must be launched folded into the launch vehicle fairing and deployed (both optics and structure) once on 


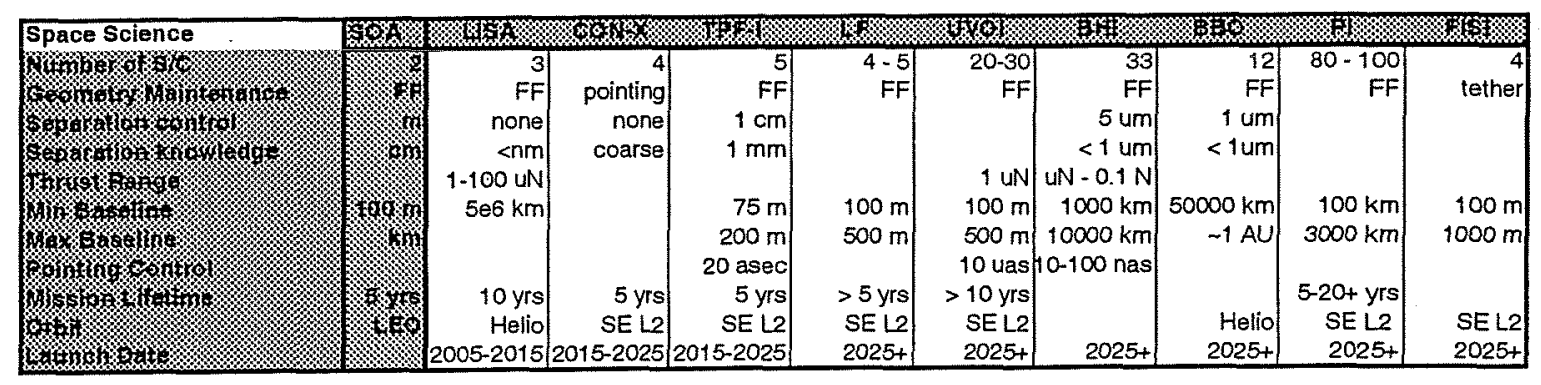

Figure 2 Key Distributed and Advanced Spacecraft System Need

orbit. Future mission studies are developing requirements for size, low mass, and stability that greatly exceed those of JWST. If these future telescope/observatory missions are to be realized we must have the capability to develop larger precision structures. This requires development of new materials with high stiffness/mass ratios, good thermal conductivity and good damping characteristics.

Strongly coupled to the size of a structure is its required stability. Stability requirement ranges from nanometers to picometers for interferometers and coronagraphs to micrometers to nanometers for very large (tens of meters) radar systems. While specific requirements for large precision structures vary with application, there is a common set of high-level areas of investment that span all applications: Structure Stability and Precision, Materials Properties and Implementation Technology.

These three areas are strongly interconnected and must be approached with a long-term, system level investment strategy. For example, materials creep and precision thermal performance in a space environment are fundamental factors in any stability model, but appropriate environment material properties (particularly at very low temperatures) have never been measured for a wide range of potentially valuable materials. A broad understanding of materials properties will be needed to develop cost effective/acceptable risk stable structures. Similarly, issues with regard to implementation technology (e.g., launch load reduction systems and trades among deployment vs assembly vs inflatabilty) factor strongly into design architectures. A comprehensive set of system-level trade studies comparing and quantifying these parameters is needed to guide investment strategies for future missions.

\subsection{CRYOGENIC AND THERMAL CONTROL SYSTEMS}

Cryogenic and thermal control systems include passive and active technologies used to cool large optical systems and thermal isolation necessary for stable operation. The stateof-the-art in this area is the sunshade and thermal isolation system currently under development to passively cool the
JWST telescope assembly. Heat switches, advanced radiators, heat pipes, and capillary pump loops are all technologies, which need to be further improved both in efficiency, size, and cost to better enable high- and lowtemperature cooling applications. The area of coolers greatly overlaps with the needs of scientific sensors.

For long wavelength (e.g., infrared and far-infrared) applications, cooled primary mirrors are an enabling capability. As shown in Figure 3, the sensitivity of Single Aperture Far Infrared (SAFIR) mission can be improved by two orders of magnitude if the telescope optics can be cooled from current the $\sim 30 \mathrm{~K}$ achievable via passive cooling to a $4 \mathrm{~K}$ telescope temperature with the addition of active cooling. Lowering the thermal background is equivalent to increasing the size of the aperture.

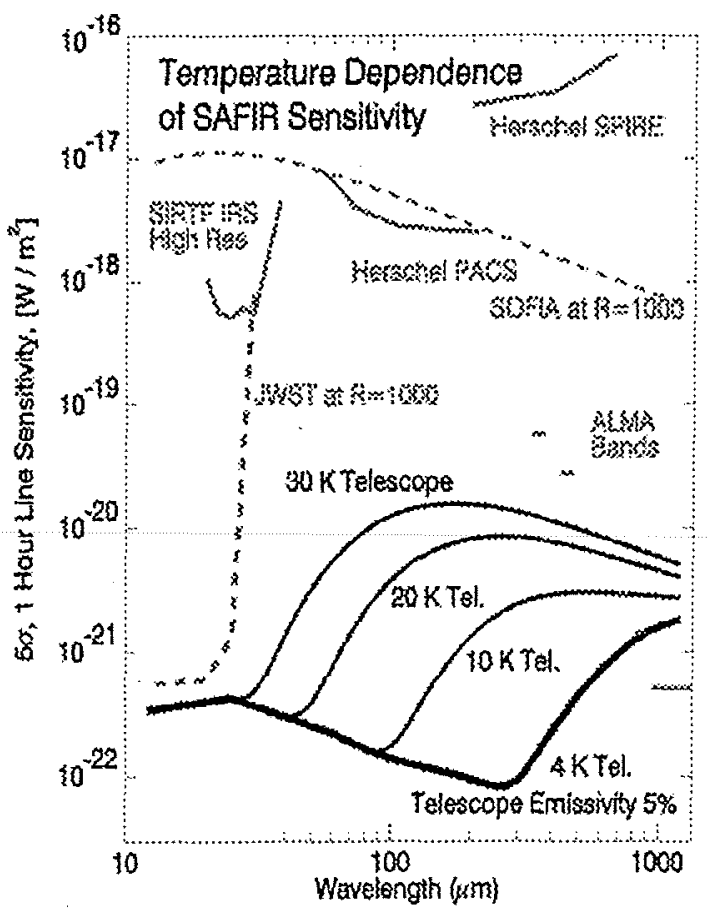

Figure 3. Effects of Cooling on SAFIR 


\subsection{INFRASTRUCTURE}

Infrastructure (both ground and space) has been identified as an ATO sub-capability because of the critical role it plays in enabling cost-effective missions. The ATO Committee addressed four key areas of the infrastructure: Workforce, Integration and Test Facilities, Systematic Performance Modeling, and In-Space Operations and Servicing.

\section{Workforce:}

The development of ever more sophisticated advanced optical systems in space requires workers with specialized training and experience. Some believe that we are already exceeding the current national capability for training such people. A solution could involve improvement in both education (schools and universities) and training (experience and apprenticeships). NASA can enable these improvements by providing input and funding to educational institutes and by facilitating intern-type experiences at NASA centers and contractor facilities.

\section{New Test Facilities:}

New facilities for thermal vacuum testing may need to be considered to execute this roadmap. Large thermal vacuum test facilities have historically been a major cost and schedule consideration for large space telescopes, and will be even more challenging for future 10-meter and larger observatories. In the past, individual missions have been responsible for modified or building new facilities even though they can often benefit multiple missions. Next generation NASA missions, such as TPF-C, Constellation$\mathrm{X}$, very large microwave apertures and SAFIR will build upon the test legacy of JWST. But, they will also have new and unique test facility requirements. NASA must decide whether use of existing facilities are sufficient or whether a new facility that can more cost-effectively accommodate these and other missions is necessary. If a new facility is developed, it will be required to maximize flexibility in the cryo-thermal system, the cryogenic distribution system, optical metrology penetration, access ports for payload installation, and vibration isolation systems to accommodate multiple future programs. Finally, the facility plan must consider programmatic and logistic factors, such as the transportation of payloads to and from the facility and program schedule impacts.

\section{Systematic Model Validation using Flight Data:}

Eventually, it is probably that future space telescopes will become simply too large to test on the ground. When this occurs, modeling tools will be required to predict in-space performance. Developing the infrastructure for very large, future systems requires as yet unplanned test and analysis of data from existing flight programs to verify analytical models and ensure that future on-orbit assembly and maintenance systems will operate as predicted. It is essential to verify that subsystem analysis tools provide adequate insight into end-item performance parameters. At the very least, additional telemetry may be required. And, engineering sensors may need to be integrated into flight systems. Additionally, early development of new analytical tools and the combination of these tools with a robust verification plan during traditional integration and testing of current flight programs will provide a high level of confidence for future development of on-orbit assembly and test programs for new missions.

\section{In-Space Observatory Servicing and Assembly:}

Future space telescopes will be complex, expensive, and many will operate in Sun-Earth L2 halo orbits. A servicing capability with a follow-on capability to assemble large optical systems in space may be enabling. But, it can only be cost-effective if it leverages in-space operations capabilities developed for other missions, specifically for lunar surface and Mars exploration missions. Such leveraging opportunities should be actively pursued, and the possibilities identified in the near future, since any decision to service or assemble a space telescope must be made early in development. SAFIR may be the logical first observatory candidate for servicing because of its timing, complexity, and potential for life extension and upgrades. Future larger aperture telescopes, such as Life Finder, are optimal candidates for on-orbit assembly because their size and mass may exceed plausible future launch vehicle size.

\subsection{CAPABILITY ROADMAP TIMELINES}

The top level capability timeline for the Advanced Telescope and Observatory Roadmap is shown in Figures 4a $\& 4$ b. It rolls up key sub-capabilities milestone readiness to support a particular mission requirement. The timeline lists strategic missions that require ATO capabilities across the top. Key capabilities that enable these missions are then shown with arrows pointing to the first mission supported. The capabilities are assumed to be required 5 years prior to a mission; that is, when the technology must be at TRL- 6 . These capabilities then align with key milestones and metrics that appear within the green banner at the time needed in the appropriate ATO sub-capability (e.g., optics). This provides a clear audit trail from missions to milestones in each of the essential technologies. Detailed subcapability and technology roadmaps showing the technology progression and sub-capability development were developed and presented to the NAS. 


\subsection{SUMMARY}

The NASA Advanced Telescope and Observatory (ATO) Capability Roadmap addresses technologies necessary for NASA to enable future space telescopes and observatories operating in all electromagnetic bands, from $\mathrm{X}$-rays to millimeter waves, and including gravity-waves.

The major technical challenges are shown in Table 3. These challenges were chosen because they enable critical missions or provide a generic capability that can enable multiple missions. Technologies like optics and wavefront sensing and control are, like detectors, critical to enabling new types of science and are the most critical technology needed for these missions. Other technologies, such as formation flying, could enable multiple longer-term missions. Challenges in the area of infrastructure were identified because of their critical importance in making missions cost-effective or programmatically viable. The dates in Table 3 indicate when the ATO determined that development investments should begin such that the capability will be ready in time to support its enabling mission.

Finally, this roadmap represents but a snapshot in time as to NASA's needs. It must be understood that these needs do evolve and change over time.: Furthermore, these needs can be dramatically impacted by any number of higher level strategic decisions, such as the development of a heavy lift launch vehicle with the ability to launch much larger aperture telescopes.

\section{REFERENCES}

NASA, "The New Age of Exploration NASA's Direction for 2005 and Beyond, February 2005.

NASA capabilities roadmap: advanced telescopes and observatories Lee D. Feinberg, Proc. SPIE Int. Soc. Opt. Eng. 5899, 589906 (2005)

Bush, George W., "Vision for Space Exploration", 14 January 2004, Washington, DC.

NASA Advanced Planning and Integration Office Roadmap

Beichman, C. A., "NASA strategic roadmap: advanced telescope searches for Earth-like planets", UV/Optical/IR Space Telescopes:Innovative Technologies and Concepts II, SPIE Proceedings Vol. 5899, In Press, SPIE, Bellingham, WA.

White, N. E., "NASA strategic roadmap: origin, evolution, structure, and density of the universe", UV/Optical/IR Space Telescopes:Innovative Technologies and Concepts II, SPIE Proceedings Vol. 5899, In Press,
SPIE, Bellingham, WA.

National Research Council, "Astronomy and Astrophysics in the New Millenium", National Academy Press, 2001, Washington, DC.

"Advanced Telescope \& Observatories Capability Roadmap," Presentation to National Research Council, 15 March 2005.

"NASA Capability Roadmaps Executive Summary", NASA Document, 22 May 2005.

\section{BIOGRAPHY}

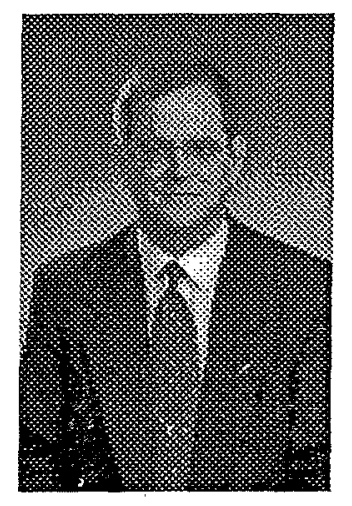

Dr. H. Philip Stahl earned his Ph.D. in Optical Sciences from the University of Arizona Optical Sciences Center in 1985. He is currently a Senior Optical Physicist at NASA MSFC where he is the JWST Optical Components Lead responsible for procurement insight/oversight for the JWST primary, secondary and tertiary mirrors; NGST Mirror Technology. Lead responsible for developing candidate primary mirror technologies; and, AMSD Technology Lead. Dr. Stahl is a leading authority in optical metrology, optical engineering, and phase-measuring interferometry. Many of the world's largest telescopes have been fabricated with the aid of high-speed and infrared phase-measuring interferometers developed by Dr. Stahl, including the Keck, VLT and Gemini telescopes.

Previously, Dr. Stahl was a Senior Staff Optical Engineer at Raytheon Danbury (formerly Hughes Danbury Optical Systems and now Goodrich Aerospace) where he was lead optical engineer on the $4 \mathrm{~m}$ Alpha/Lamp Integration program, on the Space Based Laser Program and for $4 \mathrm{~m}$ metrology capability. As President of Stahl Optical Systems Inc. he supported two microgravity experiments: the Surface Tension Driven Convection Experiment (STDCE-2) and the Droplet Combustion Experiment (DCE). Also, he was an Assistant Professor of Physics and Applied Optics at Rose-Hulman Institute of Technology, the Optical Products Manager at Breault Research Organization (BRO).

Dr. Stahl is a member of OSA and SPIE (Fellow). He received his BA in Physics and Mathematics from Wittenberg University (1979), and his MS and Ph.D. in Optical Sciences from the University of Arizona (1983 and 1985). 


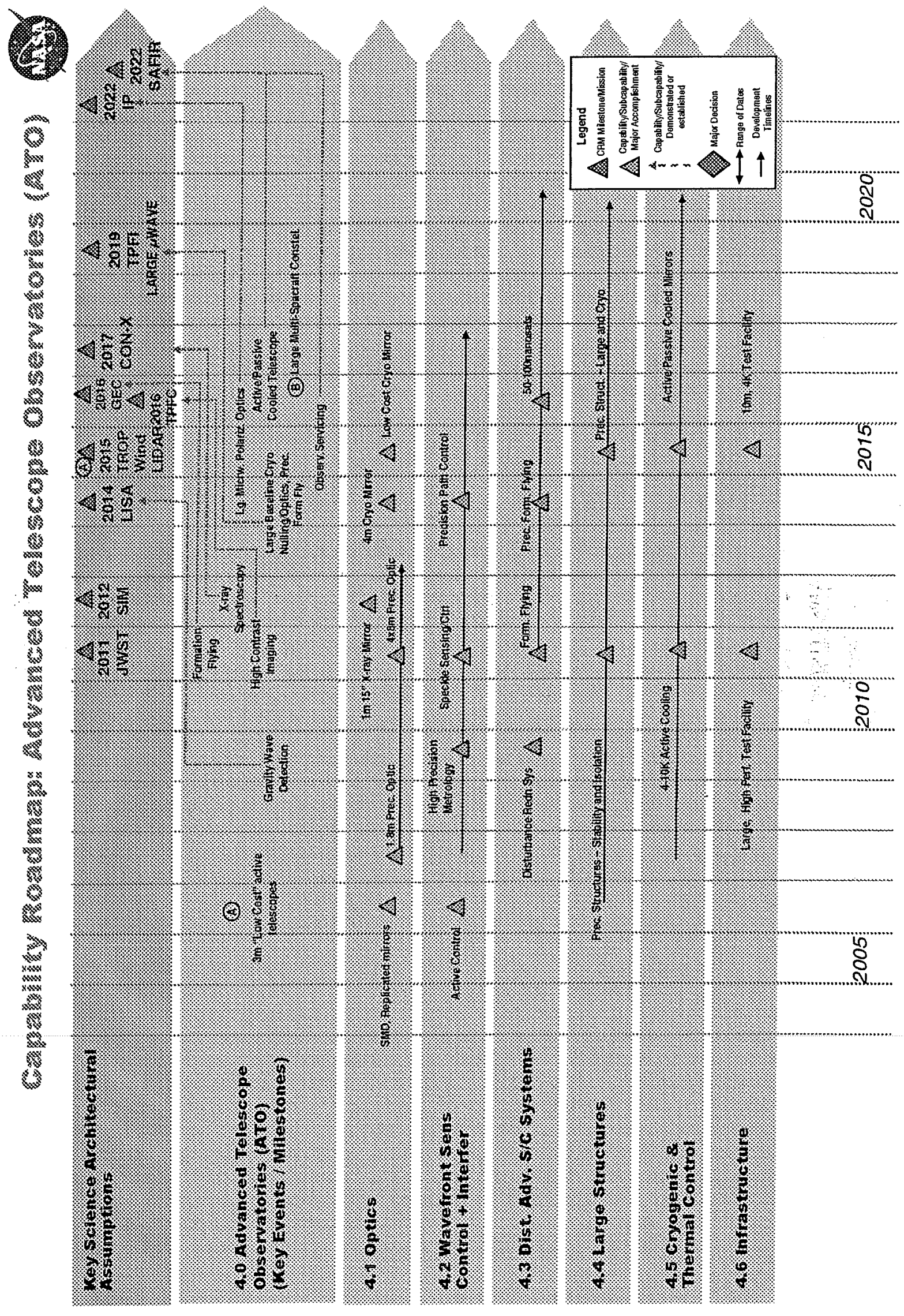

Figure 4a Capability Roadmap Timelines 


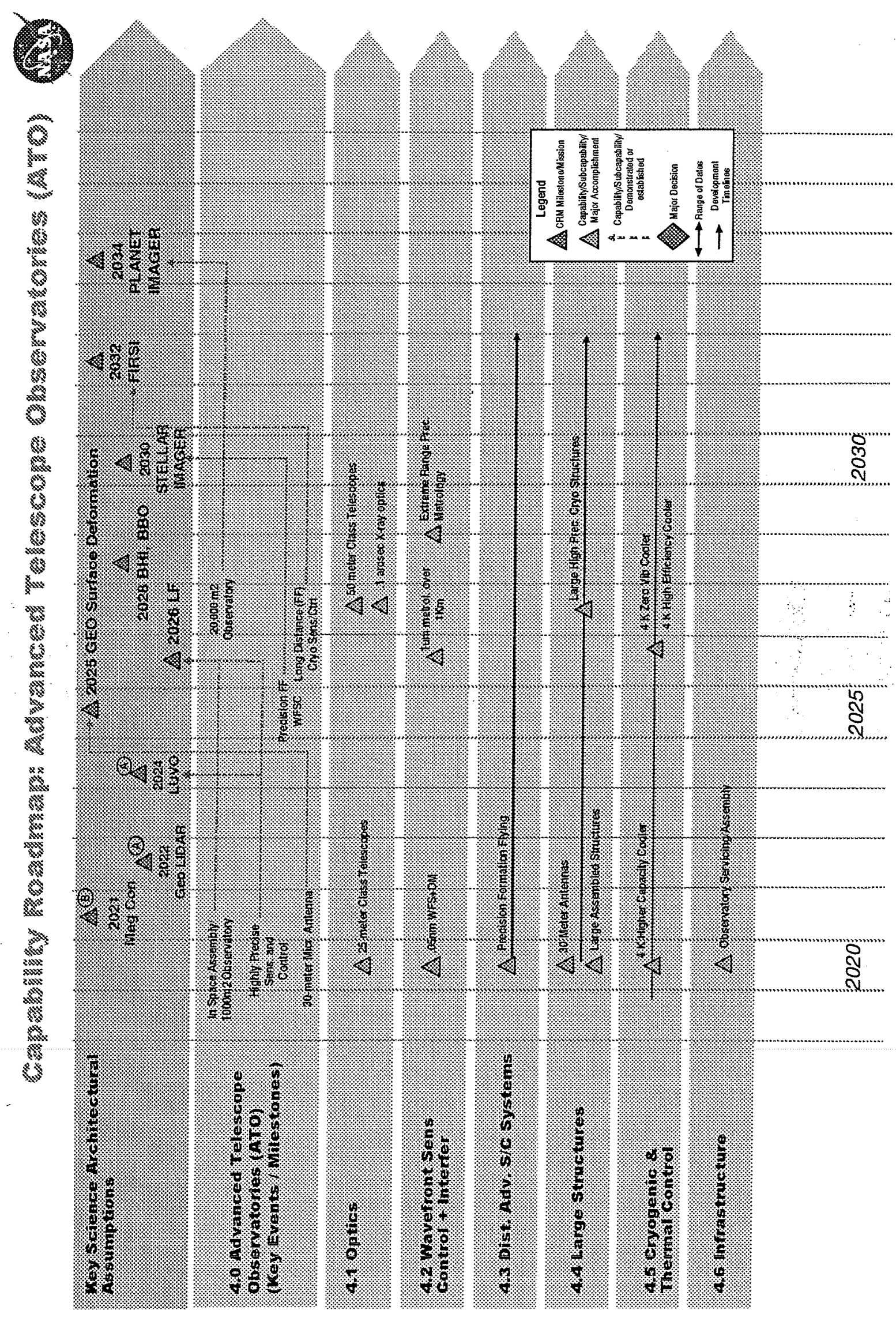

Figure 4b Capability Roadmap Timelines 
Table 3 - Major Technical Challenges

\begin{tabular}{|c|}
\hline TII \\
\hline $\begin{array}{l}\text { Very Large Precision Mirrors for TPF-C } \\
4 \times 8 \text { meter monolithic mirror }\left(<\$ 2 \mathrm{M} / \mathrm{m}^{2} \text { and }<50 \mathrm{~kg} / \mathrm{m}^{2}\right) \text {, fabricate with very small mid-spatial } \\
\text { frequency surface figure errors }(4 \mathrm{~nm} \mathrm{rms}) \text {, coating reflectance uniformity, coating polarization } \\
\text { uniformity, precision metrology for qualifying mirror specifications. }\end{array}$ \\
\hline $\begin{array}{l}\text { Low-Cost Large-Aperture, Lightweight Grazing Incidence Mirrors for Con-X } \\
\left(1.6 \times 1 \text { meter segments, } 15 \text { arc second resolution, }<\$ 0.1 \mathrm{M} / \mathrm{m}^{2},<3 \mathrm{~kg} / \mathrm{m}^{2}\right) \text {, manufacturing technology - } \\
\text { replication, etc., mirror substrate materials - thermal stability, areal density, stiffness, etc. }\end{array}$ \\
\hline $\begin{array}{l}\text { High-temporal-bandwidth wave front sensing and control (WSFC) for real-time active control of segmented } \\
\text { telescopes (LUVO, 3-meter-class low-cost telescopes). }\end{array}$ \\
\hline $\begin{array}{l}\text { High contrast speckle-reduction algorithms that achieve } 10^{10} \text { broadband contrast for TPF-C. Could include active } \\
\text { WFSC and improved occulters. }\end{array}$ \\
\hline $\begin{array}{l}\text { Formation Flying Technology Demonstrations. Roughly three quarters of long-term proposed Earth and space } \\
\text { science missions emphasized distributed and formation flight architecture. Need a sequence of formation flying } \\
\text { tests that mature these technologies in a cost-effective manner. }\end{array}$ \\
\hline $210 \%$ \\
\hline $\begin{array}{l}\text { Low-Cost } 3 \text { meter Class Mirrors } \\
\text { Manufacturing Technology - Low-cost replication enables Earth, solar, astronomy missions } \\
\text { Mirror Substrate Materials - Thermal stability, areal density, stiffness, etc. } \\
\left.\text { cryogenic mirrors for SAFIR ( } 200 \mathrm{~nm} \mathrm{rms},<\$ 0.5 \mathrm{M} / \mathrm{m}^{2} \text { and }<25 \mathrm{~kg} / \mathrm{m}^{2}\right) \\
\text { Precision Mirrors for LUVO }\left(5 \mathrm{~nm} \mathrm{~ms},<\$ 2 \mathrm{M} / \mathrm{m}^{2} \text { and }<25 \mathrm{~kg} / \mathrm{m}^{2}\right)\end{array}$ \\
\hline $\begin{array}{l}\text { Replicated Spacecraft and Formation Control. Multi-spacecraft formations are expensive and propellant } \\
\text { consumption places strict limitations on lifetime options. }\end{array}$ \\
\hline $\begin{array}{l}\text { Active/Passive Cooled Optical Systems - Combination of passive cooling techniques (like sunshields) with active } \\
\text { coolers to get } 4-10 \mathrm{~K} \text { cooling of large mirror surface area. }\end{array}$ \\
\hline $\begin{array}{l}\text { Integration and test paradigm shift from system assembly and test on the ground to final system deployment and } \\
\text { verification in space. This requires a new level of confidence in software modeling and increased complexity (e.g., } \\
\text { degrees of freedom). }\end{array}$ \\
\hline On-orbit servicing and assembly capabilities, leveraging human and in-space robotics capabilities. \\
\hline advanced nulling that will \\
\hline 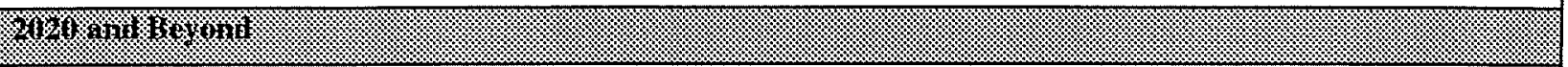 \\
\hline $\begin{array}{l}\text { Low-Cost Large-Aperture, Lightweight Grazing Incidence Mirrors for EUXO } \\
\left(8 \text { meter segments, } 0.1 \text { arc second resolution, }<\$ 1 \mathrm{~K} / \mathrm{m}^{2},<0.5 \mathrm{~kg} / \mathrm{m}^{2}\right)\end{array}$ \\
\hline $\begin{array}{l}\text { Many Spacecraft in Large Baseline Formations. Increasing the number of spacecraft complicates on-line } \\
\text { maneuver path planning, sensing and control as well as changes in the manufacturing and testing process. Large } \\
\text { separations create synchronization, sensing and communications challenges. }\end{array}$ \\
\hline
\end{tabular}

